CRYSTALLOGRAPHIC COMMUNICATIONS

ISSN 2056-9890

Received 7 June 2015

Accepted 19 June 2015

Edited by S. Parkin, University of Kentucky, USA

Keywords: crystal structure; 4-hydroxypyridin-1 ium; 3,5-dicarboxybenzoate; hydrogen

bonding; cocrystal

CCDC reference: 1407819

Supporting information: this article has supporting information at journals.iucr.org/e

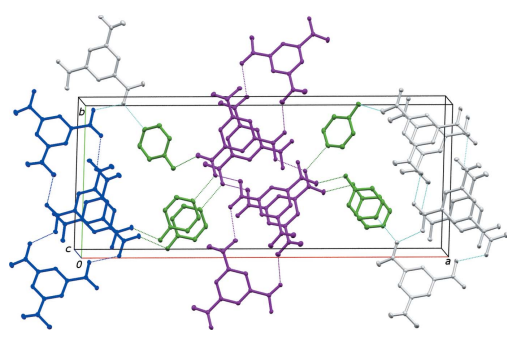

OPEN $\odot$ ACCESS

\section{Crystal structure of 4-hydroxypyridin-1-ium 3,5-dicarboxybenzoate}

\author{
Selena L. Staun and Allen G. Oliver*
}

Department of Chemistry and Biochemistry, University of Notre Dame, Notre Dame, IN 46556-5670, USA. *Correspondence e-mail: aoliver2@nd.edu

The structure of the title salt, $\mathrm{C}_{5} \mathrm{H}_{6} \mathrm{NO}^{+} \cdot \mathrm{C}_{9} \mathrm{H}_{5} \mathrm{O}_{6}{ }^{-},(\mathbf{I})$, shows that 4-hydroxypyridine has abstracted an $\mathrm{H}$ atom from benzene-1,3,5-tricarboxylic acid, yielding a pyridinium cation and carboxylate anion. The two ions form an extensive three-dimensional hydrogen-bonded network throughout the crystal. The hydrogen bonds that comprise the core of the network are considered strong, with $\mathrm{O}-\mathrm{H} \cdots \mathrm{O}$ and $\mathrm{N}-\mathrm{H} \cdots \mathrm{O}$ donor-to-acceptor distances ranging from 2.533 (2) to 2.700 (2) $\AA$. Packing is further enhanced by $\pi$-stacking of the cations and anions with like species [centroid-centroid distance = 3.6206 (13) ̊].

\section{Chemical Context}

As a study in crystal engineering utilizing hydrogen bonding between disparate molecules (Desiraju, 2003), we have been investigating the cocrystallization of various pyridine compounds with benzene carboxylic acids (Staun \& Oliver, 2012). From previous work, 4-hydroxypyridine undergoes hydrogen migration from the hydroxy $\mathrm{O}$ to the pyridine $\mathrm{N}$ atom, yielding 4-pyridone (Tyl et al., 2008). We were surprised to find that in the case of 4-hydroxypyridin-1-ium 3,5-dicarboxybenzoate, (I), an $\mathrm{H}$ atom is abstracted from one carboxylic acid group, yielding a pyridinium salt. This result allows for the hydroxy $\mathrm{O}$ and pyridine $\mathrm{N}$ atom to both act as hydrogen-bond donors, rather than the donor/acceptor situation of the 4pyridone species. These two molecules have been incorporated as linker species in metal-organic frameworks (Guo et al., 2011).<smiles>O=C([O-])c1cc(C(=O)O)cc(C(=O)O)c1</smiles><smiles>Oc1cc[nH+]cc1</smiles>

\section{Structural Commentary}

The structure of (I) shows that the 4-hydroxypyridine has abstracted an $\mathrm{H}$ atom from the benzenetricarboxylic acid, yielding a pyridinium cation and a carboxylate anion (Fig. 1). Bond distances about the pyridine ring show some localization of the bonds: $\mathrm{C} 1-\mathrm{C} 2$ and $\mathrm{C} 4-\mathrm{C} 5$ are slightly shorter than the ideal aromatic distance $[1.367$ (3) and 1.369 (3) $\AA$, respec- 


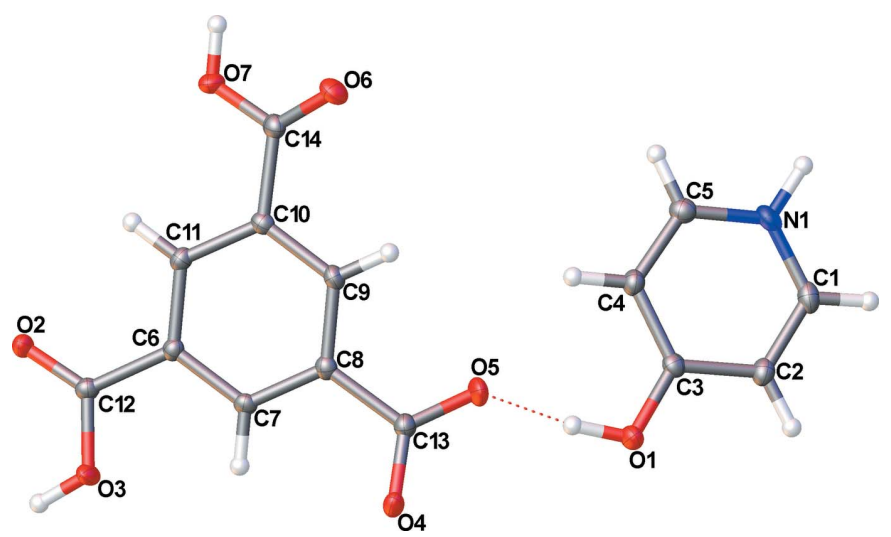

Figure 1

Labeling scheme for (I). Displacement ellipsoids are depicted at the $50 \%$ probability level. The inter-ion hydrogen bond is shown as a dashed red line.

tively, $c f .1 .390 \AA$ for an aromatic $\mathrm{C}-\mathrm{C}$ bond]. The $\mathrm{N} 1-\mathrm{C} 1$ and $\mathrm{N} 1-\mathrm{C} 5$ distances are typical for an aromatic $\mathrm{N}$ atom [1.345 (3) and 1.348 (3) ^, respectively]. The remaining bonds within the ring display typical aromatic distances $[\mathrm{C} 2-\mathrm{C} 3=$ 1.405 (3) $\AA$ and $\mathrm{C} 3-\mathrm{C} 4=1.402$ (3) $\AA$ ]. The $\mathrm{C} 3-\mathrm{O} 1$ distance of 1.326 (2) $\AA$ is typical for a hydroxy $\mathrm{O}$ atom bound to an aromatic ring. Bond angles within the pyridine ring are unexceptional.

Two of the three carboxylic acid groups show distinct singleand double-bond character $[\mathrm{C} 12-\mathrm{O} 3=1.305(3) \AA$ and $\mathrm{C} 14-\mathrm{O} 7=1.332$ (3) $\AA$; $\mathrm{C} 12-\mathrm{O} 2=1.224$ (2) $\AA$ and $\mathrm{C} 14-\mathrm{O} 6$ $=1.204(3) \AA]$. The remaining carboxylate group displays $\mathrm{C}-$ $\mathrm{O}$ bond distances that are similar to each other and indicate delocalization of the $\mathrm{C}-\mathrm{O}$ bonds $[1.268$ (3) and 1.249 (2) $\AA$ for $\mathrm{C} 13-\mathrm{O} 4$ and $\mathrm{C} 4-\mathrm{O} 5$, respectively], supporting the proposed single negative charge on the benzenetricarboxylic acid molecule. This is further supported by the presence of $\mathrm{H}$ atoms, located in a difference Fourier map, on atoms $\mathrm{O} 3$ and O7. Bond distances and angles within the benzene ring are as expected.

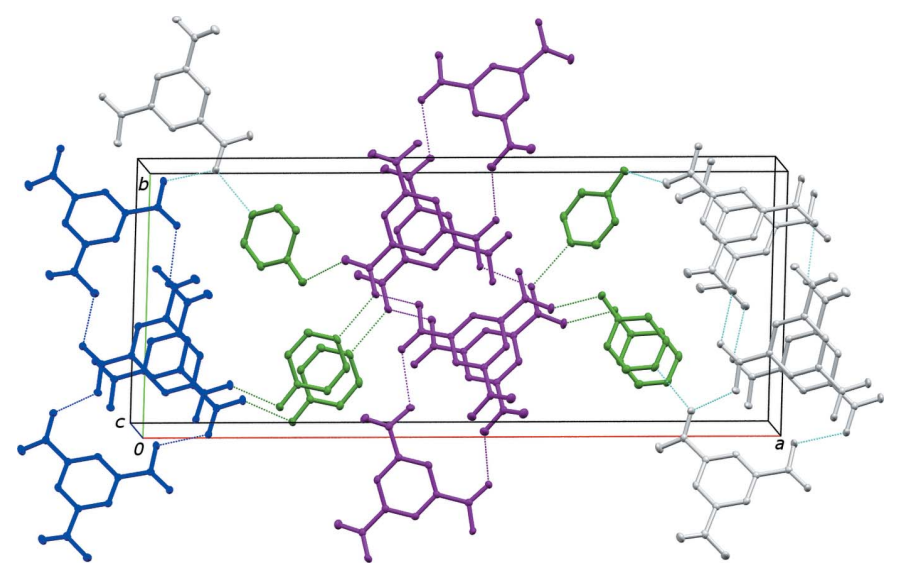

Figure 2

A view of (I) approximately along the crystallographic $c$ axis. Color code: blue represents the $R_{4}^{4}(28)$ ring, purple the $R_{6}^{6}(44)$ ring, and green the bridging 4-hydroxypyridinium cations.
Table 1

Hydrogen-bond geometry $\left(\AA{ }^{\circ}\right)$.

\begin{tabular}{lllll}
\hline$D-\mathrm{H} \cdots A$ & $D-\mathrm{H}$ & $\mathrm{H} \cdots A$ & $D \cdots A$ & $D-\mathrm{H} \cdots A$ \\
\hline $\mathrm{O} 1-\mathrm{H} 1 \mathrm{O} \cdots \mathrm{O} 5$ & $0.95(3)$ & $1.59(4)$ & $2.533(2)$ & $171(3)$ \\
$\mathrm{N} 1-\mathrm{H} 1 \mathrm{~N} \cdots 4^{\mathrm{i}}$ & $0.98(3)$ & $1.73(3)$ & $2.700(2)$ & $173(4)$ \\
$\mathrm{O} 3-\mathrm{H} 3 \mathrm{O} 4^{\mathrm{ii}}$ & $0.93(3)$ & $1.65(3)$ & $2.574(2)$ & $172(3)$ \\
$\mathrm{O} 7-\mathrm{H} 7 \mathrm{O} \cdots 2^{\mathrm{iii}}$ & $0.90(3)$ & $1.79(3)$ & $2.678(2)$ & $166(3)$ \\
\hline
\end{tabular}

Symmetry codes: (i) $-x+\frac{1}{2}, y+\frac{1}{2}, z-\frac{1}{2}$; (ii) $-x,-y, z-\frac{1}{2}$; (iii) $-x,-y+1, z+\frac{1}{2}$.

Table 2

$\pi$-stacking interactions within (I).

\begin{tabular}{lll}
\hline Interaction & $C g \cdots C g(\AA)$ & $C g \cdots \operatorname{perp}(\AA)$ \\
\hline$C g 1 \cdots C g 1^{\mathrm{i}}$ & $3.6206(13)$ & $3.4373(9)$ \\
$C g 2 \cdots C g 2^{\mathrm{i}}$ & $3.6206(13)$ & $3.3627(9)$ \\
\hline
\end{tabular}

$C g 1$ is the centroid of the 3,5-dicarboxybenzoate ring, $C g 2$ is the centroid of the 4 hydroxypyridinium ring [symmetry code: (i) $\mathrm{x}, \mathrm{y}, 1+\mathrm{z}$ ], $\mathrm{Cg} \cdots \mathrm{Cg}$ is the centroid-to-

\section{Supramolecular Features}

The local intermolecular contacts consist of the pyridinium cation forming a hydrogen bond from the hydroxy group to

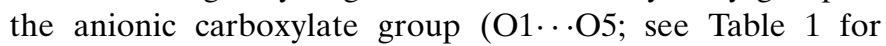
detailed contacts) and from pyridine atom N1 to carboxylate atom $\mathrm{O} 4^{\mathrm{i}}$ [symmetry code: (i) $-x+\frac{1}{2}, y+\frac{1}{2}, z-\frac{1}{2}$ ]. Carboxylic acid atoms $\mathrm{O} 3$ and $\mathrm{O} 7$ are donors for hydrogen bonds to atoms $\mathrm{O} 4^{\mathrm{ii}}$ and $\mathrm{O} 2^{\mathrm{iii}}$, respectively [symmetry codes: (ii) $-x$, $-y, z-\frac{1}{2}$; (iii) $-x,-y+1, z+\frac{1}{2}$ ]. Since these hydrogen bonds extend over several molecules, an extensive hydrogen-bonded network exists in this structure.

Pertinent features of this extended network are an $R_{4}^{4}(28)$ ring comprised of 3,5-dicarboxybenzoate ions (Fig. 2) (Bernstein et al., 1995). The carboxylic acid groups are involved in the hydrogen bonding within this ring. There is also an $R_{6}^{6}(44)$ ring of 3,5-dicarboxybenzoate ions, that incorporate a different chain of carboxylic acid groups. These rings are bridged by the 4-hydroxypyridinium cations resulting in the three-dimensional network. The hydrogen bonds within the structure are surprisingly strong, with $\mathrm{O}-\mathrm{H} \cdots \mathrm{O}$ and $\mathrm{N}-$ $\mathrm{H} \cdots \mathrm{O}$ distances ranging from $2.533(2)$ to $2.700(2) \AA$ (Table 1).

The cations and anions form homogeneous $\pi$-stacked columns parallel to the $c$ axis, that is, 4-pyridinium cations stacking with other cations and 3,5-dicarboxybenzoate anions stacking with other anions. The centroid-to-centroid distances for both the pyridinium and the dicarboxybenzoate interactions are 3.6206 (13) $\AA$, i.e. the $c$-axis spacing. The centroidto-perpendicular distances are 3.3629 (9) $\AA$ for the cation and 3.4372 (9) $\AA$ for the anion. Both measurements are within accepted $\pi-\pi$ contact ranges (see Table 2; Spek, 2009).

\section{Database Survey}

A search of the Cambridge Structural Database (CSD, Version 5.36 plus 3 updates; Groom \& Allen, 2014) for 4hydroxypyridine and benzenetricarboxylic acid gave only five 
Table 3

Experimental details.

\begin{tabular}{|c|c|}
\hline \multicolumn{2}{|l|}{ Crystal data } \\
\hline Chemical formula & $\mathrm{C}_{5} \mathrm{H}_{6} \mathrm{NO}^{+} \cdot \mathrm{C}_{9} \mathrm{H}_{5} \mathrm{O}_{6}^{-}$ \\
\hline$M_{\mathrm{r}}$ & 305.24 \\
\hline Crystal system, space group & Orthorhombic, $P n a 2_{1}$ \\
\hline Temperature $(\mathrm{K})$ & 122 \\
\hline$a, b, c(\AA)$ & $\begin{array}{l}29.3465(10), 12.2113(5), \\
\quad 3.6206(2)\end{array}$ \\
\hline$V\left(\AA^{3}\right)$ & $1297.47(10)$ \\
\hline$Z$ & 4 \\
\hline Radiation type & $\mathrm{Cu} K \alpha$ \\
\hline$\mu\left(\mathrm{mm}^{-1}\right)$ & 1.10 \\
\hline Crystal size $(\mathrm{mm})$ & $0.11 \times 0.06 \times 0.06$ \\
\hline \multicolumn{2}{|l|}{ Data collection } \\
\hline Diffractometer & Bruker APEXII \\
\hline Absorption correction & $\begin{array}{l}\text { Numerical (SADABS; Krause } e t \\
\quad \text { al., 2015) }\end{array}$ \\
\hline$T_{\min }, T_{\max }$ & $0.694,0.753$ \\
\hline $\begin{array}{l}\text { No. of measured, independent and } \\
\text { observed }[I>2 \sigma(I)] \text { reflections }\end{array}$ & $6000,2322,2172$ \\
\hline$R_{\text {int }}$ & 0.018 \\
\hline$(\sin \theta / \lambda)_{\max }\left(\AA^{-1}\right)$ & 0.614 \\
\hline \multicolumn{2}{|l|}{ Refinement } \\
\hline$R\left[F^{2}>2 \sigma\left(F^{2}\right)\right], w R\left(F^{2}\right), S$ & $0.028,0.071,1.05$ \\
\hline No. of reflections & 2322 \\
\hline No. of parameters & 213 \\
\hline No. of restraints & 1 \\
\hline H-atom treatment & $\begin{array}{l}\mathrm{H} \text { atoms treated by a mixture of } \\
\text { independent and constrained } \\
\text { refinement }\end{array}$ \\
\hline$\Delta \rho_{\max }, \Delta \rho_{\min }\left(\mathrm{e} \AA^{-3}\right)$ & $0.15,-0.19$ \\
\hline Absolute structure & $\begin{array}{l}\text { Flack } x \text { determined using } 786 \\
\text { quotients } \\
{\left[\left(I^{+}\right)-\left(I^{-}\right)\right] /\left[\left(I^{+}\right)+\left(I^{-}\right)\right]} \\
\quad(\text { Parsons } \text { et al., 2013) }\end{array}$ \\
\hline Absolute structure parameter & $0.20(8)$ \\
\hline
\end{tabular}

Computer programs: APEX2 and SAINT (Bruker 2012), SHELXS97 (Sheldrick, 2008), SHELXL2014 (Sheldrick, 2015), OLEX2 (Dolomanov et al., 2009), Mercury (Macrae et al., 2008), POVRay (Cason, 2003), publCIF (Westrip, 2010) and PLATON (Spek, 2009).

hits. In the compound that is most closely related to the title compound, namely benzene-1,3,5-tricarboxylic acid pyridin4(1H)-one (Campos-Gaxiola et al., 2014), there are three molecules of 4-pyridone present in the asymmetric unit. Benzenetricarboxylic acid and a tetrakis[(pyridin-4-yloxy)methyl]methane moiety (incorporating a 4-hydroxypyridine functionality) have been utilized in the devlopment of frameworks incorporating copper and cadmium (Guo et al., 2011).

\section{Synthesis and Crystallization}

To a solution of benzene-1,3,5-tricarboxylic acid $(0.035 \mathrm{~g}$, $1.24 \mathrm{mmol}$ ) in $\mathrm{MeOH}(3 \mathrm{ml})$ in a $20 \mathrm{ml}$ vial was added a solution of 4-hydroxypyridine $(0.0218 \mathrm{~g}, 1.77 \mathrm{mmol})$ in $\mathrm{MeOH}$ $(3 \mathrm{ml})$. The mixture was shaken vigorously, covered with perforated Parafilm and allowed to evaporate slowly over a period of $5 \mathrm{~d}$, yielding colorless rod-like crystals.

\section{Refinement}

Crystal data, data collection and structure refinement details are summarized in Table 3. Carboxylic, hydroxy, and pyridinium $\mathrm{H}$ atoms were initally located in a difference Fourier map. $\mathrm{H}$ atoms on the 4-hydroxypyridinium cation were refined freely. $\mathrm{H}$ atoms on the carboxylic acid groups were included with refined coordinates and atomic displacement parameters tied to that of the $\mathrm{O}$ atom to which they are bonded. $\mathrm{C}-\mathrm{H}$ hydrogens were included in idealized positions riding on the $\mathrm{C}$ atom to which they are bonded, with $\mathrm{C}-\mathrm{H}$ distances constrained to $0.95 \AA$ and $U_{\text {iso }}(\mathrm{H})=1.2 U_{\text {eq }}(\mathrm{C})$.

The compound is achiral, but crystallizes with a noncentrosymmetric, polar space group. The Flack $x$ parameter refined to 0.20 (8), which suggests the possibility of a small amount of inversion twinnning (Parsons et al., 2013), but the strength of the anomalous signal is very weak. We compared both a model twinned by inversion and the untwinned model, and there was no significant difference. We therefore elected to model the structure without inclusion of a twin component.

\section{Acknowledgements}

We thank the University of Notre Dame for generous support of the micro-focus source used in this project. SLS thanks the Indiana Academy of Science for a Junior Research Grant and the Henderson lab for kind donation of materials and research space.

\section{References}

Bernstein, J., Davis, R. E., Shimoni, L. \& Chang, N.-L. (1995). Angew. Chem. Int. Ed. Engl. 34, 1555-1573.

Bruker (2012). APEX2 and SAINT. Bruker-Nonius AXS Inc. Madison, Wisconsin, USA.

Campos-Gaxiola, J. J., Zamora Falcon, F., Corral Higuera, R., Höpfl, H. \& Cruz-Enríquez, A. (2014). Acta Cryst. E70, o453-o454.

Cason, C. J. (2003). POV-RAY. Persistence of Vision Ray Tracer Pty Ltd, Victoria, Australia.

Desiraju, G. R. (2003). J. Mol. Struct. 656, 5-15.

Dolomanov, O. V., Bourhis, L. J., Gildea, R. J., Howard, J. A. K. \& Puschmann, H. (2009). J. Appl. Cryst. 42, 339-341.

Groom, C. R. \& Allen, F. H. (2014). Angew. Chem. Int. Ed. 53, 662671.

Guo, J., Ma, J.-F., Liu, B., Kan, W.-Q. \& Yang, J. (2011). Cryst. Growth Des. 11, 3609-3621.

Krause, L., Herbst-Irmer, R., Sheldrick, G. M. \& Stalke, D. (2015). J. Appl. Cryst. 48, 3-10.

Macrae, C. F., Bruno, I. J., Chisholm, J. A., Edgington, P. R., McCabe, P., Pidcock, E., Rodriguez-Monge, L., Taylor, R., van de Streek, J. \& Wood, P. A. (2008). J. Appl. Cryst. 41, 466-470.

Parsons, S., Flack, H. D. \& Wagner, T. (2013). Acta Cryst. B69, 249259.

Sheldrick, G. M. (2008). Acta Cryst. A64, 112-122.

Sheldrick, G. M. (2015). Acta Cryst. C71, 3-8.

Spek, A. L. (2009). Acta Cryst. D65, 148-155.

Staun, S. L. \& Oliver, A. G. (2012). Acta Cryst. C68, o84-087.

Tyl, A., Nowak, M. \& Kusz, J. (2008). Acta Cryst. C64, o661-o664. Westrip, S. P. (2010). J. Appl. Cryst. 43, 920-925. 


\section{supporting information}

Acta Cryst. (2015). E71, 861-863 [doi:10.1107/S2056989015011780]

\section{Crystal structure of 4-hydroxypyridin-1-ium 3,5-dicarboxybenzoate}

\section{Selena L. Staun and Allen G. Oliver}

\section{Computing details}

Data collection: APEX2 (Bruker, 2012); cell refinement: SAINT (Bruker 2012); data reduction: SAINT (Bruker 2012); program(s) used to solve structure: SHELXS97 (Sheldrick, 2008); program(s) used to refine structure: SHELXL2014 (Sheldrick, 2015); molecular graphics: OLEX2 (Dolomanov et al., 2009), Mercury (Macrae et al., 2008) and POVRay (Cason, 2003); software used to prepare material for publication: publCIF (Westrip, 2010) and PLATON (Spek, 2009).

\section{4-Hydroxypyridin-1-ium 3,5dicarboxybenzoate}

Crystal data

$\mathrm{C}_{5} \mathrm{H}_{6} \mathrm{NO}^{+} \cdot \mathrm{C}_{9} \mathrm{H}_{5} \mathrm{O}_{6}{ }^{-}$

$M_{r}=305.24$

Orthorhombic, Pna $_{1}$

$a=29.3465(10) \AA$

$b=12.2113(5) \AA$

$c=3.6206(2) \AA$

$V=1297.47(10) \AA^{3}$

$Z=4$

$F(000)=632$

\section{Data collection}

Bruker APEXII diffractometer

Radiation source: Incoatec micro-focus Detector resolution: 8.33 pixels $\mathrm{mm}^{-1}$ combination of $\omega$ and $\varphi$-scans Absorption correction: numerical SADABS (Krause et al., 2015)

$T_{\min }=0.694, T_{\max }=0.753$

Refinement

Refinement on $F^{2}$

Least-squares matrix: full

$R\left[F^{2}>2 \sigma\left(F^{2}\right)\right]=0.028$

$w R\left(F^{2}\right)=0.071$

$S=1.05$

2322 reflections

213 parameters

1 restraint

Primary atom site location: structure-invariant direct methods

Secondary atom site location: difference Fourier map
$D_{\mathrm{x}}=1.563 \mathrm{Mg} \mathrm{m}^{-3}$

$\mathrm{Cu} K \alpha$ radiation, $\lambda=1.54184 \AA$

Cell parameters from 2373 reflections

$\theta=3.4-71.2^{\circ}$

$\mu=1.10 \mathrm{~mm}^{-1}$

$T=122 \mathrm{~K}$

Rod, colorless

$0.11 \times 0.06 \times 0.06 \mathrm{~mm}$

6000 measured reflections

2322 independent reflections

2172 reflections with $I>2 \sigma(I)$

$R_{\text {int }}=0.018$

$\theta_{\max }=71.2^{\circ}, \theta_{\min }=3.0^{\circ}$

$h=-27 \rightarrow 35$

$k=-13 \rightarrow 14$

$l=-4 \rightarrow 4$

Hydrogen site location: mixed

$\mathrm{H}$ atoms treated by a mixture of independent and constrained refinement

$w=1 /\left[\sigma^{2}\left(F_{\mathrm{o}}^{2}\right)+(0.0441 P)^{2}+0.1815 P\right]$ where $P=\left(F_{\mathrm{o}}^{2}+2 F_{\mathrm{c}}^{2}\right) / 3$

$(\Delta / \sigma)_{\max }=0.001$

$\Delta \rho_{\max }=0.15$ e $\AA^{-3}$

$\Delta \rho_{\min }=-0.19$ e $\AA^{-3}$

Absolute structure: Flack $x$ determined using 786 quotients $[(\mathrm{I}+)-(\mathrm{I}-)] /[(\mathrm{I}+)+(\mathrm{I}-)]$ (Parsons et al., 2013)

Absolute structure parameter: $0.20(8)$ 


\section{Special details}

Geometry. All e.s.d.'s (except the e.s.d. in the dihedral angle between two 1.s. planes) are estimated using the full covariance matrix. The cell e.s.d.'s are taken into account individually in the estimation of e.s.d.'s in distances, angles and torsion angles; correlations between e.s.d.'s in cell parameters are only used when they are defined by crystal symmetry. An approximate (isotropic) treatment of cell e.s.d.'s is used for estimating e.s.d.'s involving l.s. planes.

Fractional atomic coordinates and isotropic or equivalent isotropic displacement parameters $\left(\AA^{2}\right)$

\begin{tabular}{|c|c|c|c|c|}
\hline & $x$ & $y$ & $z$ & $U_{\text {iso }} * / U_{\text {eq }}$ \\
\hline $\mathrm{O} 1$ & $0.23997(5)$ & $0.04165(12)$ & $0.3072(5)$ & $0.0195(4)$ \\
\hline $\mathrm{H} 1 \mathrm{O}$ & $0.2112(11)$ & $0.062(3)$ & $0.405(11)$ & $0.057(11)^{*}$ \\
\hline N1 & $0.32385(6)$ & $0.30371(16)$ & $0.1767(5)$ & $0.0181(4)$ \\
\hline $\mathrm{H} 1 \mathrm{~N}$ & $0.3450(9)$ & $0.365(3)$ & $0.166(11)$ & $0.048(10)^{*}$ \\
\hline $\mathrm{C} 1$ & $0.33796(7)$ & $0.2029(2)$ & $0.0796(6)$ & $0.0186(5)$ \\
\hline $\mathrm{H} 1 \mathrm{~A}$ & 0.3679 & 0.1933 & -0.0157 & $0.022 *$ \\
\hline $\mathrm{C} 2$ & $0.30997(6)$ & $0.11410(19)$ & $0.1159(7)$ & 0.0169 (4) \\
\hline $\mathrm{H} 2 \mathrm{~A}$ & 0.3201 & 0.0433 & 0.0444 & $0.020^{*}$ \\
\hline $\mathrm{C} 3$ & $0.26602(6)$ & $0.12932(17)$ & $0.2608(6)$ & $0.0145(4)$ \\
\hline $\mathrm{C} 4$ & $0.25177(7)$ & $0.23551(18)$ & $0.3521(6)$ & $0.0160(4)$ \\
\hline $\mathrm{H} 4 \mathrm{~A}$ & 0.2218 & 0.2481 & 0.4424 & $0.019 *$ \\
\hline $\mathrm{C} 5$ & $0.28155(7)$ & $0.32085(18)$ & $0.3095(6)$ & $0.0174(4)$ \\
\hline $\mathrm{H} 5 \mathrm{~A}$ & 0.2723 & 0.3929 & 0.3741 & $0.021 *$ \\
\hline $\mathrm{O} 2$ & $-0.06609(4)$ & $0.25817(12)$ & $0.5787(5)$ & $0.0182(3)$ \\
\hline $\mathrm{O} 3$ & $-0.04180(5)$ & $0.08670(13)$ & $0.5070(5)$ & $0.0215(4)$ \\
\hline $\mathrm{H} 3 \mathrm{O}$ & $-0.0703(9)$ & $0.067(2)$ & $0.417(9)$ & $0.032 *$ \\
\hline $\mathrm{O} 4$ & $0.11707(4)$ & $-0.02696(12)$ & $0.7027(5)$ & $0.0196(4)$ \\
\hline O5 & $0.16718(4)$ & $0.10740(13)$ & $0.6111(6)$ & $0.0252(4)$ \\
\hline O6 & $0.11934(5)$ & $0.47723(13)$ & $1.1519(5)$ & $0.0220(4)$ \\
\hline $\mathrm{O} 7$ & $0.05337(5)$ & $0.53129(13)$ & $0.8992(5)$ & $0.0204(4)$ \\
\hline $\mathrm{H} 7 \mathrm{O}$ & $0.0611(8)$ & $0.598(3)$ & $0.987(9)$ & $0.031 *$ \\
\hline $\mathrm{C} 6$ & $0.01288(6)$ & $0.21569(17)$ & $0.6846(6)$ & $0.0127(4)$ \\
\hline $\mathrm{C} 7$ & $0.04618(7)$ & $0.13490(17)$ & $0.6462(6)$ & $0.0128(4)$ \\
\hline $\mathrm{H} 7 \mathrm{~A}$ & 0.0380 & 0.0638 & 0.5628 & $0.015^{*}$ \\
\hline $\mathrm{C} 8$ & $0.09153(6)$ & $0.15874(17)$ & $0.7304(6)$ & $0.0133(4)$ \\
\hline $\mathrm{C} 9$ & $0.10330(6)$ & $0.26321(18)$ & $0.8480(6)$ & $0.0142(4)$ \\
\hline H9A & 0.1341 & 0.2795 & 0.9056 & $0.017^{*}$ \\
\hline $\mathrm{C} 10$ & $0.07019(6)$ & $0.34410(18)$ & $0.8820(6)$ & $0.0131(4)$ \\
\hline $\mathrm{C} 11$ & $0.02463(6)$ & $0.32021(17)$ & $0.8012(6)$ & 0.0134 (4) \\
\hline H11A & 0.0019 & 0.3751 & 0.8261 & $0.016^{*}$ \\
\hline $\mathrm{C} 12$ & $-0.03557(6)$ & $0.18988(17)$ & $0.5860(6)$ & 0.0138 (4) \\
\hline $\mathrm{C} 13$ & $0.12794(6)$ & $0.07323(18)$ & $0.6782(6)$ & $0.0150(4)$ \\
\hline $\mathrm{C} 14$ & $0.08419(7)$ & $0.45642(18)$ & $0.9953(6)$ & $0.0156(5)$ \\
\hline
\end{tabular}

Atomic displacement parameters $\left(\AA^{2}\right)$

\begin{tabular}{lllllll}
\hline & $U^{11}$ & $U^{22}$ & $U^{33}$ & $U^{12}$ & $U^{13}$ & $U^{23}$ \\
\hline O1 & $0.0143(7)$ & $0.0151(8)$ & $0.0291(9)$ & $-0.0021(6)$ & $0.0044(7)$ & $-0.0012(7)$ \\
N1 & $0.0152(8)$ & $0.0205(10)$ & $0.0185(9)$ & $-0.0067(8)$ & $-0.0004(7)$ & $0.0002(8)$
\end{tabular}


supporting information

\begin{tabular}{lllllll} 
C1 & $0.0122(9)$ & $0.0270(12)$ & $0.0166(11)$ & $-0.0006(9)$ & $0.0006(8)$ & $0.0004(10)$ \\
C2 & $0.0142(9)$ & $0.0208(11)$ & $0.0157(10)$ & $0.0018(8)$ & $0.0008(8)$ & $-0.0017(9)$ \\
C3 & $0.0133(9)$ & $0.0166(11)$ & $0.0135(10)$ & $-0.0014(8)$ & $-0.0015(8)$ & $-0.0005(9)$ \\
C4 & $0.0132(9)$ & $0.0180(11)$ & $0.0170(10)$ & $0.0010(8)$ & $0.0016(8)$ & $-0.0006(10)$ \\
C5 & $0.0190(10)$ & $0.0171(11)$ & $0.0161(11)$ & $-0.0012(8)$ & $0.0000(8)$ & $-0.0007(9)$ \\
O2 & $0.0116(6)$ & $0.0130(7)$ & $0.0299(9)$ & $0.0009(5)$ & $-0.0012(6)$ & $0.0000(7)$ \\
O3 & $0.0124(7)$ & $0.0138(8)$ & $0.0382(11)$ & $-0.0007(6)$ & $-0.0073(7)$ & $-0.0059(7)$ \\
O4 & $0.0122(6)$ & $0.0134(7)$ & $0.0331(9)$ & $0.0017(6)$ & $0.0034(6)$ & $0.0022(7)$ \\
O5 & $0.0120(7)$ & $0.0220(9)$ & $0.0417(11)$ & $-0.0023(6)$ & $0.0085(7)$ & $-0.0032(8)$ \\
O6 & $0.0179(7)$ & $0.0204(8)$ & $0.0277(9)$ & $-0.0050(6)$ & $-0.0047(7)$ & $-0.0032(7)$ \\
O7 & $0.0179(8)$ & $0.0126(8)$ & $0.0308(10)$ & $0.0003(6)$ & $-0.0019(7)$ & $-0.0057(7)$ \\
C6 & $0.0125(8)$ & $0.0126(10)$ & $0.0130(9)$ & $0.0008(8)$ & $0.0013(7)$ & $0.0010(8)$ \\
C7 & $0.0142(8)$ & $0.0112(10)$ & $0.0129(10)$ & $-0.0014(7)$ & $0.0007(7)$ & $0.0018(8)$ \\
C8 & $0.0128(9)$ & $0.0143(10)$ & $0.0129(10)$ & $-0.0005(8)$ & $0.0009(8)$ & $0.0022(9)$ \\
C9 & $0.0111(9)$ & $0.0166(11)$ & $0.0150(10)$ & $-0.0017(8)$ & $-0.0008(8)$ & $0.0002(9)$ \\
C10 & $0.0139(9)$ & $0.0136(10)$ & $0.0117(10)$ & $-0.0017(8)$ & $0.0003(8)$ & $0.0002(8)$ \\
C11 & $0.0139(9)$ & $0.0138(10)$ & $0.0127(10)$ & $0.0024(8)$ & $0.0004(8)$ & $0.0006(8)$ \\
C12 & $0.0152(9)$ & $0.0125(10)$ & $0.0137(10)$ & $0.0000(8)$ & $-0.0002(8)$ & $0.0009(9)$ \\
C13 & $0.0129(9)$ & $0.0159(11)$ & $0.0164(10)$ & $0.0001(8)$ & $0.0000(8)$ & $-0.0007(9)$ \\
C14 & $0.0139(9)$ & $0.0168(11)$ & $0.0162(11)$ & $-0.0012(8)$ & $0.0026(9)$ & $-0.0015(9)$ \\
& & & & & & \\
\hline
\end{tabular}

Geometric parameters $\left(\AA,{ }^{o}\right)$

\begin{tabular}{llll}
\hline $\mathrm{O} 1-\mathrm{C} 3$ & $1.326(2)$ & $\mathrm{O} 5-\mathrm{C} 13$ & $1.249(2)$ \\
$\mathrm{O} 1-\mathrm{H} 1 \mathrm{O}$ & $0.95(3)$ & $\mathrm{O} 6-\mathrm{C} 14$ & $1.204(3)$ \\
$\mathrm{N} 1-\mathrm{C} 1$ & $1.345(3)$ & $\mathrm{O} 7-\mathrm{C} 14$ & $1.332(3)$ \\
$\mathrm{N} 1-\mathrm{C} 5$ & $1.348(3)$ & $\mathrm{O} 7-\mathrm{H} 7 \mathrm{O}$ & $0.90(3)$ \\
$\mathrm{N} 1-\mathrm{H} 1 \mathrm{~N}$ & $0.98(3)$ & $\mathrm{C} 6-\mathrm{C} 11$ & $1.388(3)$ \\
$\mathrm{C} 1-\mathrm{C} 2$ & $1.367(3)$ & $\mathrm{C} 6-\mathrm{C} 7$ & $1.396(3)$ \\
$\mathrm{C} 1-\mathrm{H} 1 \mathrm{~A}$ & 0.9500 & $\mathrm{C} 6-\mathrm{C} 12$ & $1.499(3)$ \\
$\mathrm{C} 2-\mathrm{C} 3$ & $1.405(3)$ & $\mathrm{C} 7-\mathrm{C} 8$ & $1.396(3)$ \\
$\mathrm{C} 2-\mathrm{H} 2 \mathrm{~A}$ & 0.9500 & $\mathrm{C} 7-\mathrm{H} 7 \mathrm{~A}$ & 0.9500 \\
$\mathrm{C} 3-\mathrm{C} 4$ & $1.402(3)$ & $\mathrm{C} 8-\mathrm{C} 9$ & $1.389(3)$ \\
$\mathrm{C} 4-\mathrm{C} 5$ & $1.369(3)$ & $\mathrm{C} 8-\mathrm{C} 13$ & $1.506(3)$ \\
$\mathrm{C} 4-\mathrm{H} 4 \mathrm{~A}$ & 0.9500 & $\mathrm{C} 9-\mathrm{C} 10$ & $1.391(3)$ \\
$\mathrm{C} 5-\mathrm{H} 5 \mathrm{~A}$ & 0.9500 & $\mathrm{C} 9-\mathrm{H} 9 \mathrm{~A}$ & 0.9500 \\
$\mathrm{O} 2-\mathrm{C} 12$ & $1.224(2)$ & $\mathrm{C} 10-\mathrm{C} 11$ & $1.399(3)$ \\
$\mathrm{O} 3-\mathrm{C} 12$ & $1.305(3)$ & $\mathrm{C} 10-\mathrm{C} 14$ & $1.489(3)$ \\
$\mathrm{O} 3-\mathrm{H} 3 \mathrm{O}$ & $0.93(3)$ & $\mathrm{C} 11-\mathrm{H} 11 \mathrm{~A}$ & \\
$\mathrm{O} 4-\mathrm{C} 13$ & $1.268(3)$ & & $119.90(19)$ \\
& & & 120.1 \\
$\mathrm{C} 3-\mathrm{O} 1-\mathrm{H} 1 \mathrm{O}$ & $111(2)$ & $\mathrm{C} 6-\mathrm{C} 7-\mathrm{C} 8$ & 120.1 \\
$\mathrm{C} 1-\mathrm{N} 1-\mathrm{C} 5$ & $121.26(19)$ & $\mathrm{C} 6-\mathrm{C} 7-\mathrm{H} 7 \mathrm{~A}$ & $119.70(18)$ \\
$\mathrm{C} 1-\mathrm{N} 1-\mathrm{H} 1 \mathrm{~N}$ & $119.9(18)$ & $\mathrm{C} 8-\mathrm{C} 7-\mathrm{H} 7 \mathrm{~A}$ & $119.95(17)$ \\
$\mathrm{C} 5-\mathrm{N} 1-\mathrm{H} 1 \mathrm{~N}$ & $118.7(18)$ & $\mathrm{C} 9-\mathrm{C} 8-\mathrm{C} 7$ & $120.29(19)$ \\
$\mathrm{N} 1-\mathrm{C} 1-\mathrm{C} 2$ & $121.04(19)$ & $\mathrm{C} 9-\mathrm{C} 8-\mathrm{C} 13$ & $120.39(18)$ \\
$\mathrm{N} 1-\mathrm{C} 1-\mathrm{H} 1 \mathrm{~A}$ & 119.5 & $\mathrm{C} 7-\mathrm{C} 8-\mathrm{C} 13$ & $\mathrm{C} 8-\mathrm{C} 9-\mathrm{C} 10$ \\
$\mathrm{C} 2-\mathrm{C} 1-\mathrm{H} 1 \mathrm{~A}$ & 119.5 & &
\end{tabular}




\begin{tabular}{|c|c|c|c|}
\hline $\mathrm{C} 1-\mathrm{C} 2-\mathrm{C} 3$ & $118.9(2)$ & $\mathrm{C} 8-\mathrm{C} 9-\mathrm{H} 9 \mathrm{~A}$ & 119.8 \\
\hline $\mathrm{C} 1-\mathrm{C} 2-\mathrm{H} 2 \mathrm{~A}$ & 120.6 & $\mathrm{C} 10-\mathrm{C} 9-\mathrm{H} 9 \mathrm{~A}$ & 119.8 \\
\hline $\mathrm{C} 3-\mathrm{C} 2-\mathrm{H} 2 \mathrm{~A}$ & 120.6 & $\mathrm{C} 9-\mathrm{C} 10-\mathrm{C} 11$ & $120.1(2)$ \\
\hline $\mathrm{O} 1-\mathrm{C} 3-\mathrm{C} 4$ & $123.01(18)$ & $\mathrm{C} 9-\mathrm{C} 10-\mathrm{C} 14$ & $119.06(17)$ \\
\hline $\mathrm{O} 1-\mathrm{C} 3-\mathrm{C} 2$ & $118.02(19)$ & $\mathrm{C} 11-\mathrm{C} 10-\mathrm{C} 14$ & $120.86(18)$ \\
\hline $\mathrm{C} 4-\mathrm{C} 3-\mathrm{C} 2$ & $118.97(19)$ & $\mathrm{C} 6-\mathrm{C} 11-\mathrm{C} 10$ & $119.51(18)$ \\
\hline $\mathrm{C} 5-\mathrm{C} 4-\mathrm{C} 3$ & $119.15(19)$ & $\mathrm{C} 6-\mathrm{C} 11-\mathrm{H} 11 \mathrm{~A}$ & 120.2 \\
\hline $\mathrm{C} 5-\mathrm{C} 4-\mathrm{H} 4 \mathrm{~A}$ & 120.4 & $\mathrm{C} 10-\mathrm{C} 11-\mathrm{H} 11 \mathrm{~A}$ & 120.2 \\
\hline $\mathrm{C} 3-\mathrm{C} 4-\mathrm{H} 4 \mathrm{~A}$ & 120.4 & $\mathrm{O} 2-\mathrm{C} 12-\mathrm{O} 3$ & $123.40(18)$ \\
\hline $\mathrm{N} 1-\mathrm{C} 5-\mathrm{C} 4$ & $120.7(2)$ & $\mathrm{O} 2-\mathrm{C} 12-\mathrm{C} 6$ & $123.77(19)$ \\
\hline $\mathrm{N} 1-\mathrm{C} 5-\mathrm{H} 5 \mathrm{~A}$ & 119.7 & $\mathrm{O} 3-\mathrm{C} 12-\mathrm{C} 6$ & $112.83(17)$ \\
\hline $\mathrm{C} 4-\mathrm{C} 5-\mathrm{H} 5 \mathrm{~A}$ & 119.7 & $\mathrm{O} 5-\mathrm{C} 13-\mathrm{O} 4$ & $124.62(19)$ \\
\hline $\mathrm{C} 12-\mathrm{O} 3-\mathrm{H} 3 \mathrm{O}$ & $117.1(18)$ & $\mathrm{O} 5-\mathrm{C} 13-\mathrm{C} 8$ & $116.56(19)$ \\
\hline $\mathrm{C} 14-\mathrm{O} 7-\mathrm{H} 7 \mathrm{O}$ & $110.8(17)$ & $\mathrm{O} 4-\mathrm{C} 13-\mathrm{C} 8$ & $118.82(17)$ \\
\hline $\mathrm{C} 11-\mathrm{C} 6-\mathrm{C} 7$ & $120.42(17)$ & $\mathrm{O} 6-\mathrm{C} 14-\mathrm{O} 7$ & $124.0(2)$ \\
\hline $\mathrm{C} 11-\mathrm{C} 6-\mathrm{C} 12$ & $120.09(17)$ & $\mathrm{O} 6-\mathrm{C} 14-\mathrm{C} 10$ & $124.07(19)$ \\
\hline $\mathrm{C} 7-\mathrm{C} 6-\mathrm{C} 12$ & $119.45(18)$ & $\mathrm{O} 7-\mathrm{C} 14-\mathrm{C} 10$ & $111.89(17)$ \\
\hline $\mathrm{C} 5-\mathrm{N} 1-\mathrm{C} 1-\mathrm{C} 2$ & $-0.7(3)$ & $\mathrm{C} 7-\mathrm{C} 6-\mathrm{C} 11-\mathrm{C} 10$ & $-0.3(3)$ \\
\hline $\mathrm{N} 1-\mathrm{C} 1-\mathrm{C} 2-\mathrm{C} 3$ & $-0.7(3)$ & $\mathrm{C} 12-\mathrm{C} 6-\mathrm{C} 11-\mathrm{C} 10$ & $-178.11(19)$ \\
\hline $\mathrm{C} 1-\mathrm{C} 2-\mathrm{C} 3-\mathrm{O} 1$ & $-177.5(2)$ & $\mathrm{C} 9-\mathrm{C} 10-\mathrm{C} 11-\mathrm{C} 6$ & $-0.5(3)$ \\
\hline $\mathrm{C} 1-\mathrm{C} 2-\mathrm{C} 3-\mathrm{C} 4$ & $2.1(3)$ & $\mathrm{C} 14-\mathrm{C} 10-\mathrm{C} 11-\mathrm{C} 6$ & $177.9(2)$ \\
\hline $\mathrm{O} 1-\mathrm{C} 3-\mathrm{C} 4-\mathrm{C} 5$ & $177.3(2)$ & $\mathrm{C} 11-\mathrm{C} 6-\mathrm{C} 12-\mathrm{O} 2$ & $4.8(4)$ \\
\hline $\mathrm{C} 2-\mathrm{C} 3-\mathrm{C} 4-\mathrm{C} 5$ & $-2.2(3)$ & $\mathrm{C} 7-\mathrm{C} 6-\mathrm{C} 12-\mathrm{O} 2$ & $-173.0(2)$ \\
\hline $\mathrm{C} 1-\mathrm{N} 1-\mathrm{C} 5-\mathrm{C} 4$ & $0.5(3)$ & $\mathrm{C} 11-\mathrm{C} 6-\mathrm{C} 12-\mathrm{O} 3$ & $-175.4(2)$ \\
\hline $\mathrm{C} 3-\mathrm{C} 4-\mathrm{C} 5-\mathrm{N} 1$ & $0.9(3)$ & $\mathrm{C} 7-\mathrm{C} 6-\mathrm{C} 12-\mathrm{O} 3$ & $6.7(3)$ \\
\hline $\mathrm{C} 11-\mathrm{C} 6-\mathrm{C} 7-\mathrm{C} 8$ & $0.9(3)$ & $\mathrm{C} 9-\mathrm{C} 8-\mathrm{C} 13-\mathrm{O} 5$ & $-25.5(3)$ \\
\hline $\mathrm{C} 12-\mathrm{C} 6-\mathrm{C} 7-\mathrm{C} 8$ & $178.7(2)$ & $\mathrm{C} 7-\mathrm{C} 8-\mathrm{C} 13-\mathrm{O} 5$ & $151.8(2)$ \\
\hline $\mathrm{C} 6-\mathrm{C} 7-\mathrm{C} 8-\mathrm{C} 9$ & $-0.7(3)$ & $\mathrm{C} 9-\mathrm{C} 8-\mathrm{C} 13-\mathrm{O} 4$ & $154.9(2)$ \\
\hline $\mathrm{C} 6-\mathrm{C} 7-\mathrm{C} 8-\mathrm{C} 13$ & $-178.04(19)$ & $\mathrm{C} 7-\mathrm{C} 8-\mathrm{C} 13-\mathrm{O} 4$ & $-27.8(3)$ \\
\hline $\mathrm{C} 7-\mathrm{C} 8-\mathrm{C} 9-\mathrm{C} 10$ & $-0.1(3)$ & $\mathrm{C} 9-\mathrm{C} 10-\mathrm{C} 14-\mathrm{O} 6$ & $-20.3(3)$ \\
\hline $\mathrm{C} 13-\mathrm{C} 8-\mathrm{C} 9-\mathrm{C} 10$ & $177.3(2)$ & $\mathrm{C} 11-\mathrm{C} 10-\mathrm{C} 14-\mathrm{O} 6$ & $161.3(2)$ \\
\hline $\mathrm{C} 8-\mathrm{C} 9-\mathrm{C} 10-\mathrm{C} 11$ & $0.7(3)$ & $\mathrm{C} 9-\mathrm{C} 10-\mathrm{C} 14-\mathrm{O} 7$ & $159.0(2)$ \\
\hline $\mathrm{C} 8-\mathrm{C} 9-\mathrm{C} 10-\mathrm{C} 14$ & $-177.74(19)$ & $\mathrm{C} 11-\mathrm{C} 10-\mathrm{C} 14-\mathrm{O} 7$ & $-19.4(3)$ \\
\hline
\end{tabular}

Hydrogen-bond geometry $\left(\AA,{ }^{\circ}\right)$

\begin{tabular}{lllll}
\hline$D-\mathrm{H} \cdots A$ & $D-\mathrm{H}$ & $\mathrm{H} \cdots A$ & $D \cdots A$ & $D-\mathrm{H} \cdots A$ \\
\hline $\mathrm{O} 1-\mathrm{H} 1 O \cdots \mathrm{O} 5$ & $0.95(3)$ & $1.59(4)$ & $2.533(2)$ & $171(3)$ \\
$\mathrm{N} 1-\mathrm{H} 1 N^{\cdots} 4^{\mathrm{i}}$ & $0.98(3)$ & $1.73(3)$ & $2.700(2)$ & $173(4)$ \\
$\mathrm{O} 3-\mathrm{H} 3 O \cdots \mathrm{O} 4^{\mathrm{ii}}$ & $0.93(3)$ & $1.65(3)$ & $2.574(2)$ & $172(3)$ \\
$\mathrm{O} 7-\mathrm{H} 7 O \cdots \mathrm{O} 2^{\mathrm{iii}}$ & $0.90(3)$ & $1.79(3)$ & $2.678(2)$ & $166(3)$ \\
\hline
\end{tabular}

Symmetry codes: (i) $-x+1 / 2, y+1 / 2, z-1 / 2$; (ii) $-x,-y, z-1 / 2$; (iii) $-x,-y+1, z+1 / 2$. 